\title{
VIOLENCIAS EN LA ESCUELA: PROTAGONISMO, GÉNERO Y DESIGUALDAD
}

\author{
VIOLENCE IN SCHOOL: PROTAGONISM, \\ GENDER, AND INEQUALITY
}

\author{
Concepción Fernández Villanueva ${ }^{1}$ \\ Joyce Mary Adam²,*
}

\begin{abstract}
RESUMEN: El trabajo aborda las diferencias de género en violencia en la escuela desde una perspectiva interaccionista. Se realiza un análisis de incidentes de violencia ocurridos en dos escuelas de la periferia de São Paulo (Brasil) relatados en entrevistas de grupo a jóvenes escolares de ambos sexos. A pesar de que la investigación haber localizado protagonismos de violencia de alumnas, las mujeres son menos protagonistas y más víctimas que los varones y las diferencias se acentúan en la violencia dirigida contra la institución escolar. Los argumentos explicativos son similares aunque los varones resaltan la rivalidad contra la escuela y las mujeres la rivalidad interpersonal.
\end{abstract}

Palabras-clave: Violencia. Escuela. Género. Desigualdad.

ABSTRACT: This article analyses gender differences in school violence from an interactionist perspective. It presents a qualitative analysis of violent incidents occurred in two schools in the outskirts of São Paulo (Brazil), collected through group interviews with male and female young scholars. Despite the fact that the investigation has located protagonisms of the female students violence, women in general are less protagonists and more victims than men and those differences are accentuated in the violence directed against the school institution. Explanatory arguments are similar although men emphasize rivalry against school institution and women interpersonal rivalry.

Keywords: School. Violence. Gender. Inequalities.

1.Universidad Complutense de Madrid - Departamento de Psicología Social - Madrid, España.

2.Universidade Estadual Paulista Júlio de Mesquita Filho - Departamento de Educação - Instituto de Biociências Rio Claro (SP), Brasil

*Autora correspondiente: joyce.adam@unesp.br

Dossiê organizado por: Joyce Mary Adam e Débora Cristina Fonseca 


\section{Introducción}

Un marco psicosociológico interaccionista entiende la violencia en el seno de la interacción, en el contexto de las relaciones interpersonales mediadas por el poder, las condiciones de posibilidad de los individuos y los grupos, los condicionantes estructurales de las situaciones en las que se producen y de las personas que causan los daños o sufren sus consecuencias. (FERNANDEZ VILLANUEVA, 2007) Los agresores y las víctimas no son individuos en interacción desconectados de la identidad social y de las interpretaciones que la sociedad hace de sus acciones, son miembros de categorías sociales y participan del valor e importancia social que la sociedad les otorga como parte de dichas categorías. Por ello, además de los antecedentes y los motivos de las acciones, tampoco se deben descuidar las ganancias que consiguen los agresores frente a las víctimas después de los actos de violencia. Cada uno de dichos actos, más que el resultado de los rasgos psicológicos de las personas, es una consecuencia de los conflictos sociales. Cuando se aborda la diferencia de género en la violencia escolar conviene tener claro el contexto de desigualdad de género en el que se produce y su papel en el sostenimiento de la estructura de poder que mantiene la desigualdad.

Un modelo interaccionista de la violencia de jóvenes enfatiza la dimensión estratégica, de utilidad para conseguir determinados objetivos o para evitar determinadas consecuencias en la interacción entre personas o grupos. Para entenderla en esa perspectiva se identifican cuatro factores: la identidad, la estructura de las relaciones grupales, los valores que legitiman la violencia y las representaciones imaginarias de agresores y víctimas (FERNANDEZ VILLANUEVA et al., 1998; FERNANDEZ VILLANUEVA, 2010).

La violencia como estrategia de interacción se pone de relieve en cada vez mayor número de contextos, desde lo micro social a lo estructural. Fischer y Evers (2011), en su estudio sobre parejas de adultos, ponen de relieve que el enojo y la ira no suelen ser simples manifestaciones emocionales de desagrado o frustración, sino intentos de forzar cambios en las otras personas. Peets y Kikas (2006) resaltan este objetivo estratégico en su estudio sobre violencia entre adolescentes varones. Las bandas de jóvenes violentos tienen una indudable dimensión estratégica identitária, afirmativa o defensiva, según señalan Martín Álvarez y Oropeza (2008), y Feixa, Porzio y Recio (2006). La violencia puede ser funcional para el liderazgo de jóvenes en contextos urbanos (WAASDORP et al., 2013). El vandalismo y destrozo de bienes urbanos en las revueltas de grandes ciudades europeas protagonizadas por jóvenes son, en definitiva, estrategias de respuesta a reales o imaginarias ofensas a la identidad (CICCHELI et al., 2006). Pero esta violencia de adolescentes o jóvenes es menos tolerada en las chicas que en los varones (MORENO et al., 2012).

La visión estructural de la violencia de género insiste en sus objetivos dominadores (FERNANDEZ VILLANUEVA, 2004). Tanto los grandes incidentes con resultados de muerte o lesiones graves como los pequeños micro machismos operan como una estrategia al servicio del mantenimiento de la desigualdad, mantienen y refuerzan la estructura patriarcal y los roles tradicionales de género. Incluso algunas agresiones de mujeres contra otras mujeres ejercen una función paralela a la violencia machista, muestran intenciones semejantes, de mantenimiento de los estereotipos establecidos, ya que las mujeres más tradicionales expresan cierto tipo de agresividad (PARROTT; GALLAGHER, 2008) o incluso agresión física (REIDY et al. 2009) contra otras mujeres que no se ajustan al rol tradicional femenino.

Las explicaciones interaccionistas y estratégicas de la violencia escolar son, asimismo, muy consistentes y cada vez más reconocidas y extendidas. Debarbieux y Blaya (2010) interpretan la violencia escolar como un tipo de relaciones interpersonales, establecido a lo largo de años, en las que impera la “incivilidad", los insultos, groserías humillaciones, la coerción, así como la banalización de la violencia. Es decir, la existencia de un cierto "clima" negativo en el que la violencia es útil. Astor (1998) destaca la percepción por parte de los alumnos de que viven un ambiente injusto en el que las reglas se aplican arbitrariamente o 
en el que observan negligencias o actitudes injustas de los profesores a las cuales responden con sus actos violentos. Algunos autores como Abramovay y Rua (2002), García y Madriaza (2006) y Gonçalves y Spósito (2002) destacan las cuestiones organizativas, en particular la calidad de la interacción, como un factor importante en las situaciones de conflicto y violencia entre los participantes del contexto escolar. Platero (2008) interpreta el acoso sexual masculino desde las intenciones de dominación machista y el acoso homofóbico y como una estrategia de represión de la homosexualidad y de refuerzo de las normas género tradicionales. Velasco y Álvarez-González (2015) muestran que las chicas desarrollan menos comportamientos de violencia reactiva (respuesta a actos percibidos como injustos o dañinos) y también de violencia instrumental (actos destinados a conseguir un objetivo) y que la violencia reactiva es más castigada cuando la ejercen chicas. Por ejemplo, los chicos solo son disciplinados cuando sus agresiones son intensas, lo cual parece claramente relacionado con una estereotipia de roles de género que tiende a reprimir más claramente la violencia de las mujeres. Como señalan, Buelga, Cava y Musitu (2012), las chicas necesitan rechazar la violencia para mantener una buena imagen por parte del profesorado $y$, muy probablemente, por parte de sus propios compañeros y compañeras.

En las explicaciones de estos resultados adquiere un gran relieve la interpretación de la distribución del poder entre los diversos actores escolares, los profesores y autoridades escolares (agentes institucionales), los escolares y sus compañeros y la percepción de justicia o injusticia de las normas. Por ello, las explicaciones sobre el origen y función de los incidentes de violencia son explicaciones evaluativas. Esta perspectiva no puede olvidar ni las intenciones con las que se realizan los actos ni las consecuencias que se derivan de cada uno de ellos. Cada acto de violencia tiene una dimensión sociopolítica, entendiendo como tal la posición o situación frente al poder en la interacción. Las intenciones tratan de modificar el poder interpersonal o político de los agresores y las consecuencias de la violencia modifican en realidad el poder interpersonal o político de las víctimas.

El protagonismo de las mujeres en la violencia escolar se ha investigados desde una perspectiva comparativa con los varones, con lo cual ha quedado en cierto modo invisibilizadas sus condiciones de posibilidad, sus diferentes condicionantes de situación y de contexto y, por ello, algunas de sus más importantes razones y explicaciones.

Considerando el sexo de los participantes, los chicos se ven involucrados en mayor medida que las chicas en los insultos o motes con connotaciones sexuales [...], propagan más rumores en torno a la reputación sexual de otros compañeros [...] y, también, participan más del acoso de aquellos que no cumplen los códigos de género que rigen el comportamiento masculino o femenino [...]). Por el contrario, comparadas con los chicos, las chicas participan más del acoso de aquellas personas por las que sienten algún tipo de celos [...] (NAVARRO OLIVAS, 2009, p. 151).

La adolescencia es un periodo especialmente problemático en la construcción de la identidad y en el se ponen en juego varios elementos qué se relacionan con el uso de la violencia: en primer lugar la, necesidad de separarse defenderse del entorno familiar y la relevancia del contexto de los iguales en el que necesariamente se construye una nueva manera de nombrarse y situarse. En segundo lugar, la importancia de la dimensión "grupo" como factor de refuerzo de la identidad y de reconstrucción simbólica de la misma. En tercer lugar, la construcción de la identidad de género que establece una forma más estable los rasgos aprendidos en la socialización infantil así como los estereotipos de género y las categorizaciones sociales que marcan a los hombres y a las mujeres. La educación secundaria es un lugar donde se reconstruyen y refuerzan las identidades de género de los adolescentes y dónde comienzan a aparecer los rasgos de la vida social de los adolescentes que dan un enorme relieve a la enjuiciamiento de las instituciones en las que participan 
Nos interesa el protagonismo y el victimismo de los y las adolescentes escolares de secundaria en función de sus posiciones de poder y sus dinámicas de relación. Por ello, vamos más allá de los rasgos, hasta las identificaciones de género y las representaciones de las normas de la escuela, las formas de interacción diferentes o la dinámica de las relaciones interpersonales dentro de ella.

Las mujeres ha sido agentes de la violencia en todos los contextos y especialmente en el contexto político, cuando su uso se ha democratizado, es decir, cuando las sociedades les han permitido participar en estos importantes procesos en los que se desencadena la violencia, como las guerras, el terrorismo o los procesos de exclusión y marginación de la vida social. Los motivos y los objetivos de la conducta violenta han resultado muy similares en la violencia masculina y femenina. Las razones han sido ideológicas, de legitimación y, sobre todo, de posibilidad (FERNANDEZ VILLANUEVA, 2011).

En este trabajo nos planteamos los siguientes objetivos: a) visibilizar los incidentes de violencia en que los chicos y las chicas han sido protagonistas o víctimas, tanto dentro del contexto escolar como fuera de él; b) describir las "lógicas" de esa violencia, los motivos y justificaciones que aparecen en su discurso; y c) relacionar los incidentes de violencia y sus consecuencias con las dimensiones de contexto escolar mediatizadas por el género.

\section{Metodología}

\section{Diseño}

Para lograr el objetivo de captar las condiciones de la interacción en que se produce la violencia se optó por las entrevistas de grupo repetidas (RITCHIE; LEWIS, 2003). Este procedimiento permite una cierta integración entre los miembros de los grupos y a la vez, una revisión y argumentación de la información colectiva suministrada. Lo cual favorece la expresión matizada de experiencias, perspectivas y argumentaciones. Todo ello aboca a una mayor contextualización de la información que se obtiene y permite captar de algún modo la experiencia cotidiana y natural de los participantes, desde una perspectiva compartida y colectiva.

\section{Participantes}

La muestra se compone de dos grupos de adolescentes entre 12 y 17 años asistentes a dos escuelas una de enseñanza fundamental, ciclo 2 y una de enseñanza media (escuela secundaria), en la ciudad brasileña de Rio Claro, de 160.000 habitantes localizada en el interior del Estado de São Paulo. Las escuelas, de similares características, estaban situadas en la periferia de la ciudad, en una zona de población de clase media baja y baja y son escuelas públicas. La escuela secundaria tenía aproximadamente 780 estudiantes y la mayoría de ellos provienen de barrios remotos que aún no tienen una escuela secundaria. La escuela de enseñanza fundamental tenia aproximadamente 800 estudiantes de $5^{\circ}$ a $8^{\circ}$ grado, la mayoría residía en barrios cercanos a la escuela. Los grupos, pues eran homogéneos en cuanto a su procedencia social y sus características sociodemográficas. Se realizaron en total 20 entrevistas a grupos con una similar proporción de chicos y chicas. De ellas, 10 en cada una de las escuelas, 5 a jóvenes protagonistas de actos violentos (V) y otras 5 a jóvenes no protagonistas de actos violentos (NV). La clasificación de los dos grupos se estableció según el criterio local, de la propia escuela, basado en su conocimiento de la participación de los jóvenes en actos de violencia. Cada grupo estaba compuesto por entre 6 y 8 personas, con al menos dos miembros de cada género en cada uno de ellos. Las entrevistas han sido realizadas con el acuerdo de los participantes, cuya identidad 
ha sido debidamente resguardada y protegida, manteniendo las directrices éticas de respeto y anonimato de la información obtenida. En la presentación de los discursos, las preguntas de los investigadores se marcarán con la letra I.

Herramientas de producción y análisis de la información

Las entrevistas semiestructuradas versaron sobre el conocimiento y explicación de incidentes violentos conocidos o vividos por los participantes, empezando por los ocurridos en la ciudad o los lugares de ocio y finalizando por los producidos dentro de la escuela. Se analiza el discurso tomando como eje los incidentes relatados, recogiendo todos aquellos en que se hacía referencia a algún acto de violencia tanto vivida como presenciada. Posteriormente se analizan los incidentes en los que aparecen diferencias de género. Para ello se utilizó como herramienta de codificación el programa Atlas.ti 6.2, resultando de él una serie de categorías referidas al género, como el protagonismo y el tipo de incidentes en que participaron y otras referidas a los motivos argumentos y explicaciones de los mismos ofrecidas por los propios actores.

\section{Entre la sociedad y la escuela: Genero Poder y Protagonismo en la Violencia}

Se ha hecho un recuento de la cantidad de incidentes que han aparecido en el discurso. Pero la cuantificación de estos incidentes no tiene un carácter representativo ya que nuestra muestra es pequeña para ser representativa y al mismo tiempo muy rica para realizar un análisis de la lógica y el sentido de las violencias ejercidas en el contexto escolar. No obstante, tiene un alto poder descriptivo ya que aparece muy claro incluso en estos datos simples la cuantía de la participación de género y los diversos contextos en los que se ejerce o se sufre violencia.

Los 53 incidentes de diversa gravedad en que los entrevistados fueron víctimas o protagonistas indican una alta dosis de violencia, tanto protagonizada como sufrida, y no sólo en el contexto escolar, sino en el espacio de ocio y también en la familia. Una proporción relativamente importante de chicas y chicos entrevistados ejerció violencia contra la institución, contra sus compañeros y, en algunas ocasiones, en el contexto extraescolar, dentro de grupos de ocio o bandas de jóvenes que operaban en la calle. Fueron también víctimas de violencia importante, tanto por parte de profesores o personal de la escuela, como por personas de la familia, y también en algunos casos, por la policía. Asimismo, fueron testigos de otros muchos incidentes producidos en su calle, su barrio o su escuela, lo cual confirma que en el contexto en el que estos estudiantes vivían se produce una importante cantidad de inseguridad, conflictos y agresiones.

Llama la atención la relativa gran cantidad de incidentes relatados, incluso en el grupo de jóvenes llamados no violentos (NV). Si en el grupo de violentos (V) se relatan 20 incidentes de protagonismos (en los que los jóvenes son agresores), en el grupo de NV se relatan 13. Lo cual revela que estamos en un contexto altamente conflictivo. Asimismo, se muestra la continuidad entre un grupo y otro, la ausencia de diferencias radicales. Pero también hay bastante victimismo si tenemos en cuenta que relatan 26 incidentes en que han sido víctimas, que se reparten casi por igual entre los "violentos" (13) y los "no violentos" (12).

Se destaca la victimización de las mujeres sobre todo en el grupo de los NV, en el que se relatan 11 incidentes, lo cual contrata con la victimización de los varones en el mismo grupo en el que se relata solo un incidente. Por otra parte, las mujeres del grupo NV mantienen un relativamente importante protagonismo, ya que participan en 7 , si bien de tipo leve. 
Tomados en su conjunto, tanto dentro como fuera de contexto escolar, los datos muestran un evidente protagonismo masculino que dobla al femenino (21/10). Y un evidente victimismo femenino que casi triplica al masculino (19/7). No obstante, debemos subrayar la importancia de los 10 incidentes en los que las chicas han sido protagonistas.

Tabla 1. Agresores y víctimas según lugar.

\begin{tabular}{cccc}
\hline Número de incidentes & Escuela & Sociedad & Total \\
\hline Mujeres agresoras & 9 & 1 & 10 \\
\hline Mujeres víctimas & 9 & 10 & 19 \\
\hline Varones agresores & 13 & 8 & 21 \\
\hline Varones víctimas & 4 & 3 & 7 \\
\hline Total & 35 & 22 & 57 \\
\hline
\end{tabular}

Dentro del contexto escolar, el protagonismo masculino no es tan acentuado, aunque muestra el mismo patrón de género, que acentúa el protagonismo masculino (13/9) pero, sobre todo, acentúa el victimismo femenino (9/4).

El primer "rasgo" destacable en los resultados y que enfatiza la importancia del contexto social externo y personal de los grupos entrevistados es la continuidad entre la violencia en la escuela y en la calle, especialmente en los incidentes de los varones, varios de los cuales se repiten y continúan fuera de la escuela. Los incidentes relatados por las mujeres también muestran una cierta relación entre la violencia protagonizada en la escuela, la familia y, en menor medida, en la calle. En el caso de los varones, hay una cierta conexión de la violencia ejercida en la escuela con la participación en bandas que actúan en la calle, incluso con bandas delincuentes. Por ello, los chicos fueron, en mayor medida que las chicas, agredidos o buscados por la policía, incluso buscados dentro del recinto escolar. Estos ámbitos de extensión de la violencia nos muestran una escuela conectada con otras partes de la sociedad en las cuales los alumnos y alumnas tienen un nivel diferente de participación y actividad. Las chicas interaccionan más con las familias y los chicos con el ámbito extra familiar y es en esos ámbitos donde se muestran sus diferencias. El nivel de organización de los chicos es mayor que el de las chicas. Aquellos se organizan más frecuentemente en grupos, mientras que éstas actúan en mayor medida desarticuladas, fuera de grupos, de forma individual, todo lo cual es consecuente con la mayor gravedad de los incidentes protagonizados por varones. La conexión entre la violencia dentro y fuera del contexto escolar, así como la diferente vinculación de género a ella, se muestra de forma paralela en otros estudios (GIL VILLA, 2010; ELJACH, 2011).

Una descripción más detallada de la violencia propiamente escolar nos permite clasificar los incidentes en dos categorías: los que se producen entre iguales (alumnos) y los que se producen contra el personal o contra el edificio el mobiliario (vandalismo).

La violencia de los varones se distribuye casi por igual entre la dirigida contra los iguales, la institución escolar y los profesores, Hay algunos incidentes graves de violencia de chicos contra profesores, así como incidentes de vandalismo. Por ejemplo, romper cristales, poner una bomba, insultar 
a una profesora, dar una torta a otra profesora (si bien se relata cómo no intencional) y tirar una piedra al director.

La violencia de las chicas se destina en su inmensa mayoría al ámbito interpersonal, de los iguales, principalmente a otras compañeras, con la sola excepción de un incidente en grupo contra el mobiliario escolar, en que rompieron una puerta y otro que alcanzó a una profesora accidentalmente. Por lo tanto, la violencia de los varones tiene una mayor variabilidad en su destino, se dirige a mayor número de objetivos, es más amplia y también de mayor gravedad, más importante cuando se dirige a la institución, ámbito de poder superior. Este resultado es coherente con la mayor accesibilidad masculina a la agencialidad política.

Respecto a la violencia entre iguales, los incidentes más frecuentes son los que producen entre alumnos varones, después los que se producen entre alumnas, en tercer lugar, los de varones que agreden a mujeres y, en último lugar, los de mujeres que agreden a varones, que se producen muy raramente. Entre varones se producen típicos enfrentamientos de rivalidad individual o de grupo, así como violencia defensiva personal o de los amigos. En las mujeres también se producen los enfrentamientos de rivalidad y defensa, aunque son algo menos frecuentes.

Por el lado del victimismo, el número de incidentes relatados en que los chicos son víctimas de los profesores es muy escaso. Los chicos son víctimas, en primer lugar, de los compañeros, después de los profesores y muy raramente, de sus compañeras. Por el contrario, las chicas son víctimas, en primer lugar, de los profesores, en segundo lugar, de los compañeros individualmente o en grupo, y, finalmente, de otras compañeras (individualmente o en grupo).

Parece muy significativo que se relatan seis incidentes contra cuatro mujeres, en los que son prejuiciadas, excluidas marginadas o golpeadas. En dos de ellos las agresoras son compañeras, pero, en la mayoría, los agresores son varones en grupo. Los motivos son el rechazo a algún defecto físico, a una forma de vestir, a una opción sexual diferente o a una forma de ser (ser torpe, por ejemplo). Se trata de una violencia discriminatoria y de exclusión por características bien físicas o psicológicas. En algún caso, se trata de una violencia explicitada como "de castigo".. La asimetría de poder entre chicos y chicas dentro del ámbito interpersonal en la escuela explica hacia dónde se dirige predominantemente cada tipo de violencia.

\section{Sentido, Lógicas, y Representaciones Imaginarias de La Violencia Escolar}

Las dimensiones que confieren el "sentido" y ayudan a comprender los actos son: la identidad, la estructura de relaciones grupales, la ideología (argumentario legitimador) y las "representaciones imaginarias" de las situaciones y de las personas elegidas como víctimas. Estas dimensiones se presentarán interactivamente en relación entre sí porque están entrelazadas, teniendo en cuenta que el presente imaginario conforma las identidades y las relaciones grupales. Los indicios en los que se basan las representaciones imaginarias pueden ser de una importancia real muy escasa. Sin embargo, pueden resultar insoportables para los individuos o los grupos. Se trata en ocasiones de actos simbólicos (las miradas, la demostración simbólica de poder o de superioridad o inferioridad) que son interpretados como indicadores de falta de respeto y generan violencia inmediata, como lo ilustra el siguiente discurso:

Alumno: Se te queda mirando a una chica, se queda mirándola de nuevo, se nota que el chico quiera algo (de ella). Ahí comienza. (V) 
En los incidentes producidos entre iguales lo más frecuente especialmente en las chicas es la rivalidad por la "posesión "de un lugar, un recurso, la valoración o el respeto frente a los compañeros o los amigos:

Alumna: Una vez me peleé. Estaba en el cuarto curso. Porque (yo) era amiga de la prima de ella (la contrincante) y ésta quería estar cerca de la prima, entonces se sentó en mi sitio; yo llegué, le pedí que me dejases el sitio, ella no quiso, yo la pegué y se quedó llorando (V).

Los celos, la frustración o el temor al abandono o la perdida de una amistad pueden desencadenar la violencia por motivos nimios y ser el origen de peleas, ataques personales y pequeñas agresiones.

Alumna: Hay una colega que tiene celos de mi cuando voy con otras chicas. Entonces discutimos, peleamos, es un jaleo. Entonces ella hace un infierno de mi vida con las demás. ¿Que hace ella? Alumna: Miente, por ejemplo, llega y me dice: J., fulana habló tal y tal sobre ti. Entonces yo pregunto a la otra si es verdad (eso) o no y la otra me dice que no (es verdad) Ahí ya va a exigir una justificación a la chica. Es así. (NV)

La defensa del honor propio o de familiares desencadena violencia para restablecer el "respeto" tras insulto o menosprecio de la persona o sus familiares.

La mezcla entre la violencia funcional para conseguir un deseo o recurso y el castigo a quienes no se han comportado con la lealtad esperada o amenazan la identidad personal no es infrecuente como muestra el caso siguiente:

Alumna: (Yo) estaba saliendo con un chico el sábado pasado y cuando me despedí de él mi casi mejor amiga le pidió que saliera con ella. Entonces empezó el jaleo, yo discutí con ella, le di un golpe en la cara diciéndole que era una falsa (NV)

Detrás de los incidentes hay una interpretación imaginaria de las víctimas como amenazantes, peligrosas o intolerables lo que da lugar a la justificación motivada de respuesta agresiva. Un simple insulto a un familiar puede desencadenar una agresión física. Del mismo modo, una mirada puede ser interpretada como un desafío o una amenaza "insoportable" que comienza una espiral de actos de violencia.

La grupalidad es otro de los factores más influyentes en toda la acción social de los jóvenes. La presencia y la importancia social de los adolescentes depende de su comparación y diferenciación con otros y la definición de su identidad se hace dependiendo de las definiciones de los otros grupos de jóvenes y sus identidades sociales. Por ello, la violencia entre bandas o estilos juveniles, tan característica de los contextos de ocio y de la calle, tiene su correspondencia en la institución escolar. Los incidentes motivados por el restablecimiento de la identidad de grupo amenazada o degradada o para defender a un miembro de un grupo de las amenazas de otro son relativamente frecuentes, como se destaca en la siguiente declaración:

Alumno: Muchas veces por seguridad. Alguien que es un "macho", si decide acompañar a una persona que es su amiga no va a dejar que nadie la pegue. Unos se ayudan a otros. (NV)

Pero el acoso es un proceso grupal, y no se entiende sin esa dimensión. Es el resultado de procesos de marginación, exclusión o dominación o entre grupos que se basa en la distribución de poder según la cual unos dominan otros discriminan y otros sufren. Por ello, ya se acepta con mucha 
Tabla 2. Incidentes y explicaciones: varones y mujeres.

Alumno: Pusieron una bomba. Fue el año pasado, ¿no es así, señora?

\section{Mujeres/incidente y explicaciones}

Alumna: Una vez di un golpe en la puerta de ahí abajo, había un montón de personas y se rompió. La profesora me vio dándole una patada, pero no fui yo sola la que derrumbó la puerta entera, pero ella me vio a mí, entiendes? Me echaron la culpa, lógico! Fui a juicio, respondí de los hechos, tuve que pagar otra puerta. Pero no voy a apagar otra puerta, no fui la única que la rompió y tampoco voy a decir quien más la rompió, pero no voy a pagar la puerta. Si ellos descubren quien fue... pero de mi boca no va a salir. Tuve que ir a juicio. (V)

¿Pero por qué pusieron una bomba?

Alumno: Para enfrentarse.

Alumno: Todo el mundo quería interclases. El director decía que iba a haber interclases. Eso era mejor.

Alumno: Una revuelta de los chicos.

Alumno: Cuando no hubo interclases todo el mundo se rebeló

\section{$¿$ Entonces iba a haber interclases pero finalmente no hubo?}

Alumno: Ellos tomaron nuestro dinero. Tomaron nuestro dinero para las interclases y no lo devolvieron.

Alumno: Comenzaron a dar las clases. Después de las vacaciones de Julio ya no las dieron nunca más. (V)

Incidente de apedreamiento de ventanas

¿Porque pensáis que las apedrearon?

Alumno: No procuraban mejoras para la gente, no se puede hablar.

Alumno: Se podía jugar a la pelota y (luego) ya no dejaron más. (V)
¿Por qué pensáis que los alumnos hacen eso?

Alumna: Algunos toman drogas y se vuelven locos.

Alumna: Creo que alguien vio un duende en la puerta y decidió pegar una patada

Alumna: Para llamar la atención

Alumna: Pero no estoy de acuerdo en romper las cosas por eso

Alumna: Es un momento de locura, a veces cualquiera tiene un momento de locura, ¿no? (NV) 
frecuencia que la intervención se debe enfocar desde la perspectiva de los grupos de iguales y no desde la perspectiva de los agresores y las víctimas individuales (SALMIVALLI, 2009). Como destacan las siguientes alumnas:

\begin{abstract}
Alumna:Yo tenía miedo de ir a la escuela porque en la escuela ellas me amenazaban, decían que me iban a pegar y yo me moría de miedo de ir a la escuela. Desde entonces me quedé con un trauma; ¿después pasó, sabes? Alumna: Las chicas en la calle se metían conmigo, decían que me iban a pegar, que yo era no sé qué [...] Pero nunca llegaron a pegarme. Y mi hermana pequeña también $[\ldots]$, se muere de miedo de ir a la escuela $[\ldots]$ yo tengo que ir porque las chicas la quieren pegar, porque ella es pequeña, acaba de entrar en el quinto curso y ellas quieren demostrar su poder, [...] tengo que ver lo que sucede y las chicas me amenazan con pegarme a mí también (NV)
\end{abstract}

Alumna: Le pedimos la galleta y no nos la quiso dar; entonces (nosotras) la íbamos a pegar y quitarle la galleta, entonces J. dijo: deja a la chica si no queréis que os pegue. Después estuvimos molestándola y finalmente, a la salida yo pensaba que la iba a pegar, pero al final fue ella la que me pegó. (V)

Subrayamos estos dos incidentes por ser similares a los procesos típicos de acoso que se suelen describir entre los varones: amenazas a individuos con menos poder, estrategias para conseguir objetos o recursos, estrategias de dominación, exclusión y marginación.

La violencia contra la institución, ya sea contra sus miembros o contra los bienes o el mobiliario escolar entra también en la lógica de la rivalidad identitária. Se justifica en la defensa de la dignidad de los alumnos frente a los profesores o las autoridades (DUBET, 2004). Se argumenta como un intento de restablecer un cierto orden o una justicia adecuada, cuando no se puede establecer por otros medios.

En este tipo de incidentes es donde se muestra una diferencia de género más significativa. Como señalamos anteriormente, son menos frecuentes en las chicas, pero su significado es claramente diferente, ya que muestra intenciones y funcionalidad distinta en línea con las expectativas de identidad masculina y femenina y con las posiciones de poder correspondientes a unos y otras.

Analizamos dos ejemplos de violencias graves relacionadas con el género y el contexto escolar: una chica rompe una puerta estando con otros compañeros y compañeras. Un grupo de chicos ponen una bomba en un aula. Y otros grupos de chicos apedrean las ventanas. Los incidentes "masculinos" son interpretados como una forma de influir en la institución tras una percepción de injusticia de sus normas. El desajuste entre el funcionamiento escolar y las demandas de los alumnos y la frustración de expectativas legitima la colocación de una bomba en el interior del edificio y el apedreamiento de cristales. En este último acto se expresa, además, la falta de compromiso con las promesas de mejora y la incapacidad de dialogo con la institución. Sean o no ciertas, son explicaciones de intencionalidad "política". Sin embargo, el incidente protagonizado principalmente por una chica, aunque fuese motivado por un malestar "escolar" no está redactado en términos de su intencionalidad "política" contra la escuela; es explicado en términos psicológicos, producto del consumo de drogas o de un momento de locura. (Tabla 2)

Otros relatos sobre incidentes no protagonizados directamente, sino presenciados o conocidos, y siempre de chicos, nos remiten al mismo discurso, la rebelión, la rivalidad con la institución, a veces sostenida en el tiempo, como un proceso cíclico de enfrentamiento identitário. "Es por culpa de los profesores, si el profesor dice algo, la gente responde. Ahí comienza el ciclo” [...] (V). Se considera que esta situación descrita resulta en la exclusión y rechazo de la institución por parte de los alumnos: 
Alumno: habia mucha rebelión, rompian todo, habia pocos alumnos, se rompía todo. ¿Que tipo de rebelión? Alumno: Pegar, romper todo, el tipo se rebela contra la dirección y en ocasiones recibe tres advertencias y se le expulsa. (V)

Así, parece que la violencia puede ser vivida por los jóvenes escolares como necesaria o compulsoria y justificada, como una forma inevitable de responder frente a las amenazas de la identidad. La violencia como defensa identitária legitimada aparece en las chicas referida a incidentes de rivalidad individuales, como resolver los conflictos por un objeto, un asiento, un lugar o una actividad. Pero está ausente de las justificaciones del enfrentamiento contra la escuela. Las diferencias en la legitimación de los diferentes actos de violencia, puede conducir a la inhibición por parte de las mujeres de estos actos y, lo que nos parece aún más significativo, a su ocultación o falta de denuncia de los actos de violencia de la institución, que constituyen las razones legitimadoras de los protagonistas varones. En el ejemplo siguiente, se muestran dos reacciones muy distintas ante una situación "similar". Ante una violencia del director, considerada en ambos casos por la víctima como inaceptable, el chico responde con una pedrada sobre el director. La chica argumenta una actitud protectora hacia el director y finalmente no denuncia para evitar que este pierda su cargo. Este resultado, que se repite en los incidentes extraescolares, por ejemplo, en la familia, apunta a la mayor impunidad de la violencia de la institución escolar contra las mujeres.

Alumno: Cuenta cuando peleaste con el inspector. Alumno: Fue con el director. ¿El director te pegó? ¿Cual fue el motivo de la pelea? ¿ El director pegó (a alguien) aquí en la escuela? Alumno: Nosotros éramos pequeños aun. Ese insulto al director (le llamó pinguço). Yo entraba en el servicio, el (director) pensó que había sido yo y me pegó. Yo Salí y le di una pedrada. (NV) Alumna: Una vez hubo una pelea con pieles de plátano. El director vio una chica tirando, la agarro y la pego una bofetada. ¿Y que hizo ella? Se volvió contra él y el la llevó a su despacho y le pidió disculpas diciendo que fue un error pegarle. La chica dijo: no es así. Voy a ir a la policía a denunciar [...] Pero ella no fue a la policía porque si no el perdería su cargo (NV).

La pregunta que se deriva de estos resultados es si la violencia de los chicos no está más destinada que la de las chicas a conseguir o establecer el poder o la agencia como actores políticos. Al menos el umbral de respuesta a la violencia (percibida) por parte de la institución es más bajo. Habría que ahondar mucho más en esta cuestión si se quiere conocer mejor e intervenir en la violencia contra la escuela. La violencia "política" es prioritariamente legitimada por los varones, ya que son actores más influyentes, y se les considera tradicionalmente más acostumbrados y preparados para incidir y tomar decisiones que transformen la vida pública. La violencia entendida como una acción destinada a la transformación de lo público se puede considerar (sea o no condenable) como un acto político.

\section{Discusion}

La comparación con los datos de otros estudios no resulta fácil, dadas las diferencias de perspectiva. Aun así, los resultados en varios países coinciden con los nuestros y apuntan a un mayor protagonismo masculino, es decir, una mayor implicación de los varones como agresores (HOFF et al., 2009; SHUTE et al., 2008; VELASCO; ÁLVAREZ-GONZÁLEZ, 2015) No aparece tan clara la mayor victimización de las mujeres. La constatación de las diferencias suele conducir a una explicación que alude a las "diferencias de género en conducta agresiva”, que es una de las constantes más establecidas y fuertes en la psicología, o a factores más psicosociales, como los estereotipos o las identificaciones de género (GINI; POZZOLI, 2006; 
NAVARRO OLIVAS, 2009; RICHARDSON; HAMMOCK, 2007). No obstante, no aparecen con claridad otras dimensiones sociales, de contexto, de situación y, en definitiva, de interacción, como las que hemos señalado, que puedan explican el protagonismo, inhibición o recepción de actos de violencia.

Desde una perspectiva interaccionista debemos atender a las consecuencias personales y sociales de la violencia, tanto la ejercida como la sufrida. Las diferencias entre varones y mujeres en la legitimación de los diferentes actos de violencia pueden conducir a la inhibición por parte de las mujeres de su violencia contra la institución y, lo que nos parece aún más significativo, a la ocultación o falta de denuncia de los actos de violencia de la institución cuando ellas son víctimas.

Muchos actos de violencia tienen consecuencias positivas, al menos a corto plazo y si se trata de actos no graves (como es el caso de la resolución de conflictos por el espacio o los recursos mediante agresiones entre personas). Así, tanto chicos como chicas consiguen que no les quiten su sitio, que no agredan a sus amigos, que no les desafíen o insulten sus compañeros. De este modo, la estrategia violenta les resulta útil y la pueden conservar y utilizar mientras sea así. Pero el contexto normativo establece sanciones y consecuencias interpersonales a la comisión de actos violentos. Los indicios de nuestras investigaciones, apuntan a una mayor sanción de la violencia femenina: un caso similar el de poner una bomba en el interior de la escuela (chicos) y romper una puerta (chica) acarreó peores consecuencias para la chica, ya que fue llevada al juzgado y denunciada y en el caso de la bomba no se imputó a nadie legalmente. La penalización de la violencia según el género en la escuela debería ser investigada con mayor profundidad. Pero, independientemente de las sanciones legales o de la institución, hay siempre una traslación a la imagen de los agresores, una consecuencia en la identidad y la representación social de los agresores que tiende a deslegitimar y sancionar fuertemente la violencia de las mujeres. La violencia de adolescentes o jóvenes es menos tolerada en las chicas (MORENO et al., 2012). Según Fischer y Evers (2011) en los contextos de relación tradicional, las mujeres inhiben más su enojo que los varones y se atreven menos a expresarlo, debido a las expectativas de valoración social negativas. En el contexto escolar ocurre lo mismo: Buelga, Cava y Musitu (2012) muestran la estereotipia de género que obliga a estas a rechazar la violencia para mantener una imagen aceptable de cara a la institución y a sus propios compañeros. Así pues, para que la agresividad de las mujeres fuese igualmente útil o al menos no tuviese mayores consecuencias negativas que la de los varones es necesario que exista un contexto igualitario, que es muy infrecuente.

Las mujeres protagonistas se encuentran con una paradoja. Por un lado, pueden conseguir ciertos objetivos mediante la violencia, pero, por otro, la violencia no es coherente con la definición aceptada de la identidad femenina: por mucho que les sea útil, no es lo que se espera de ellas, ni tampoco lo que ellas esperan o consideran adecuado para sí mismas. Se encuentran tanto con la incomprensión externa como con la autoculpabilización. Los costes de ser violentas son mayores para las mujeres. Pero los beneficios sin duda existen: defenderse de otros, conseguir objetivos o simplemente reivindicar posiciones de poder en las relaciones interpersonales y frente a las instituciones. La violencia puede ser una estrategia para resituarse frente a los varones, como ya parece que lo es para defenderse de las mujeres rivales. Por ello es de esperar que la violencia aumente en la medida que las identidades femeninas desarrollen la consecución de logros y potencien la necesidad de ser agentes activos de todos los procesos sociales.

Ser víctima también tiene sus costes y consecuencias. Las consecuencias son, además del dolor o la humillación, la inseguridad en el centro escolar, el miedo, la desprotección. Dado que las mujeres son las peor paradas en el victimismo, estas consecuencias serán en ellas más graves. Y dada su diferencial disponibilidad para enfrentarse con la institución, más violencia recibida por parte de ésta quedará impune. 


\section{Conclusiones}

Nuestras conclusiones apuntan a dos cuestiones básicas: por un lado, explicamos las diferencias de género en el protagonismo y victimismo que podríamos resumir en las siguientes

1. La diferencia de género en la violencia escolar muestra una continuidad con la explicación del resto de las violencias en la vida social. Tanto en uno como en otro lugar, los chicos son más protagonistas y las mujeres, más víctimas. Este aspecto se relaciona con las formas de interacción diferencial Tanto en un contexto como el otro las chicas interaccionan más con los más próximos y los chicos con el ámbito más amplio

2. En consecuencia, dentro de la escuela la violencia que ejercen los varones se dirige a ámbitos más amplios que la de las mujeres (es más frecuente en los chicos la dirigida contra la institución escolar y los profesores y en las chicas la violencia contra los iguales, sobre todo las de su mismo sexo. Confluye con este rasgo el nivel de organización de los chicos que actúan más frecuentemente en grupos, de forma colectiva.

3. Sobre, la violencia que sufren, aparte de que el victimismo de los chicos es menor, muchos de los daños recibidos por ellos proceden de los iguales y algunos, de la institución. Pero las chicas reciben más daños (en proporción a la violencia ejercida por ellas) de la institución y los superiores que los varones,

4. En cuanto a las consecuencias de la violencia y considerando de nuevo que la violencia masculina suele ser más importante y más grave, existen diferencias en la legitimación de los actos cometidos, ya que todo apunta a que la violencia de las mujeres está menos entendida y menos legitimada.

5. Aunque muchos actos de violencia tienen consecuencias positivas, al menos a corto plazo, es decir, resulta instrumental para defenderse de las distintas amenazas de compañeros, profesores o la institución, los indicios de nuestras investigaciones apuntan a una mayor sanción de la violencia femenina, los incidentes masculinos al producirse en mayor cantidad en grupo presentan una relativamente mayor dificultad para ser sancionados. Y, Por otro lado, la identidad de los hombres y las mujeres que agreden sufre de forma diferente al ser la violencia masculina más aceptable que la violencia femenina.

Nuestra perspectiva se interesa por visibilizar y entender la violencia de las mujeres. Partimos de dos supuestos que proceden de la psicología feminista: a) la uniformidad básica de los rasgos de los hombres y las mujeres y la no creencia en la polaridad de identidades; b) los rasgos tienen una determinada finalidad de acción, están asociados a la consecución de determinados niveles de poder y participación social. Por lo tanto, más que los rasgos en sí mismos interesan su función, su destino final en la interacción interpersonal.

La cantidad de incidentes y, sobre todo, el destino de la violencia y su "función "dentro de la interacción sigue apuntando a diferencias relevantes y consistentes con la posición de poder y actividad correspondientes a los géneros. Hay incidentes comunes a los varones y las mujeres y otros más específicos. Lo común es la rivalidad dentro del mismo género. Lo específico son dos cuestiones: la violencia contra el otro género, que es más frecuente de varones a mujeres que a la inversa y la violencia contra la institución, que es más frecuente y más grave en varones. Este último tipo de violencia puede ser considerada como política, ya que los jóvenes escolares construyen sus propias estructuras de sentido y sus expectativas sobre la institución que chocan con la realidad vigente, las presiones sociales y los límites. Es ahí donde el individuo intenta 
incidir e en el desarrollo de lo político y actúa como sujeto político defendiéndose y justificando la violencia contra la escuela. En este punto conectamos de nuevo con las identidades de género, que reconducen la acción femenina al mundo de lo interpersonal, y restringen la agencia social y política. Podemos afirmar que cierta violencia masculina más frecuente en la escuela tiene un sentido político que legitima más a los varones que a las mujeres. Del mismo modo que la agresión no es afín a la identificación femenina, la violencia en la escuela, sobre todo la que tiene una dimensión más institucional, es coherente también con la masculinidad hegemónica, que vincula la acción masculina al mundo de lo extrapersonal.

La explicación de las diferencias en términos de rasgos de conducta o de personalidad tiende a polarizar los rasgos y a construir una imagen de varón agresivo-mujer pacífica que minimiza la importancia del protagonismo de las mujeres en todas las situaciones en las que se produce un conflicto. Desde una perspectiva interaccionista, que parte de una similitud en los fundamentos de la subjetividad y de las potencialidades individuales, son las condiciones del contexto las que ayudan a explicar las diferencias de género en el protagonismo y el victimismo de la violencia.

Esta investigación contribuye al reciente debate sobre la igualdad y la diferencia de género. Por una parte, es abrumadora la evidencia de protagonismo masculino en la violencia. Por otra, las revisiones críticas sobre movimientos políticos caricaturizan y pretenden invalidar la literatura feminista que establece una igualdad básica en las potencialidades psicológicas de los géneros incluyendo la violencia. Así pues, visualizar la violencia femenina es entrar en un debate complejo y tiene importantes costes políticos. La escasa participación femenina en violencia se transforma en una imagen de pasividad, masoquismo, de falta de agencia de las mujeres que no se corresponde con su identidad deseada desde la identificación igualitaria. Pero, en contrapartida, establecer que la mujer se implica en actos violentos que se producen en la sociedad significa reconocer su carácter "negativo" y problemático. Desde las identificaciones tradicionales de género es incongruente aceptar la igualdad en este rasgo, pero desde las nuevas identidades tampoco resulta deseable. Así como los demás rasgos "masculinos" como la agencia, la iniciativa, y las actitudes necesarias para participar en la vida pública son deseados y reconocidos por y para las mujeres, existe una resistencia a aceptar y reconocer la violencia, ya que, al contrario de las demás actitudes, está vinculada a las guerras, los conflictos y la muerte.

No queremos decir con esto que hombres y mujeres se impliquen por igual y que su violencia sea equivalente. Por el contrario, la visibilización de las diferencias relacionadas con los diversos contextos y condicionantes, nos permite aportar dos conclusiones aceptables e integrables en las nuevas identificaciones femeninas igualitarias. Por una parte, afirmamos la existencia de diferencias en la conducta violenta, pero visualizamos una acción que puede ser crucial en el futuro de la igualdad de género y no negamos que las mujeres puedan ser capaces, del mismo modo que los hombres de llevar a cabo las acciones necesarias para progresar en igualdad, aunque impliquen violencia. Por otra parte, esta constatación nos lleva a considerar los factores estructurales por los que las mujeres y los hombres ejercen determinados tipos de acciones: las posiciones y poderes en que están situados unos y otras y que permiten o inhiben ciertas manifestaciones de agencia, entre otras, la violencia. La implicación de estas conclusiones es transformadora, puesto que de ella se deriva que las violencias femenina y masculina podrían ser más similares en la medida en que el poder y las oportunidades de género lo fueran.

\section{Contribución de las Autoras}

Cuestionamiento y conceptualización; Vilanueva CF, Adam JM. Investigación; Vilanueva CF, Adam JM. Analizando; Vilanueva CF, Adam JM. Escritura; Vilanueva CF, Adam JM. 


\section{REFERENCIAS}

ABRAMOVAY, M.; RUA, M. G. Violencias nas Escolas. Paris: Unesco, 2002.

ASTOR, R. A. Moral reasoning about school violence: informational assumptions about harm in school sub contexts. Educational Psychologist, v. 33, n. 4, p. 207-221, 1998. https://doi.org/10.1207/s15326985ep3304_5

BUELGA, S.; CAVA, M. J; MUSITU, G. Reputación social, ajuste psicosocial y victimización entre adolescentes en el contexto escolar. Anales de Psicología, v. 28, n. 1, p. 180-187, 2012.

CICCHELI, V.; GALLAND, O.; DE MAILLARD, J.; MISSET, S. Enquête sur les violences urbaines. Centre d’analyse strategique. Departement Institution and Societé. Gobierno de Francia, 2006.

DEBARBIEUX, E.; BLAYA, C. Sociología y violencia escolar: un enfoque contextual In: ORTEGA, R.x (coord.) Agresividad injustificada, bullying y violencia escolar. Madrid Alianza, 2010, p. 355-383

DUBET, F. A. O que é uma escola justa? Sâo Paulo. Cadernos de Pesquisa, v. 34, n. 123, p. 539-555, 2004.

ELJACH, S. Violencia escolar en América Latina y el Caribe: Superficie y fondo. Oficina Regional para América Latina y el Caribe Panamá. República de Panamá: Unicef, 2011.

FEIXA, C. (dir.); PORZIO, L.; RECIO, C. (coords.) Jóvenes “latinos” en Barcelona. Espacio público y cultura urbana. Barcelona: Anthropos Editorial y Ayuntamiento de Barcelona, 2006.

FERNÁNDEZ VILLANUEVA, C. Violencia contra las mujeres una visión estructural. Intervención psicosocial, v. 13, n. 2, p. 155-164, 2004.

FERNÁNDEZ VILLANUEVA, C. Violencia y agresiones: pinceladas para una psicología social interaccionista. In: ROMAY, J. (ed.) Perspectivas y retrospectivas de la psicología social en los albores del siglo XXI. Biblioteca Nueva, 2007, p. 163-170

FERNÁNDEZ VILLANUEVA, C. Quatro dimensões explicativas da violência de jovens. In: ADAM DE PAULA E SILVA, J. M.; SALLES, L. M. F. Jovens, Violência e Escola: Um Desafio Contemporâneo. São Paulo: Ed. Unesp Cultura acadêmica, 2010, p. 103-125.

FERNÁNDEZ VILLANUEVA, C. Ni almas bellas ni guerreros justos. Mujeres implicadas en violencia política. Cuestiones de género: de la igualdad y la diferencia, 2011, pp. 75-97.

FERNÁNDEZ VILLANUEVA, C. et al. Jóvenes violentos: causas psicosociológicas de la violencia en grupo. Barcelona: Icaria, 1998.

FISCHER, A. H.; EVERS, C. The social costs and benefits of anger as a function of gender and relationship context. Sex Roles, v. 65, n. 1-2, p. 23-34, 2011. https://doi.org/10.1007/s11199-011-9956-x

GARCIA, M.; MADRIAZA, P. Estudio cualitativo de los determinantes de la violencia escolar en Chile. Estudos em Psicologia, v. 11, n. 3, p. 247-256, 2006. ISSN 1678-4669. https://doi.org/10.1590/S1413-294X2006000300001

GINI, G.; POZZOLI, T. The role of masculinity in children's bullying. Sex Roles, v. 54, p. 585-588, 2006. https://doi. org/10.1007/s11199-006-9015-1

GONÇALVES, L. A.; SPÓSITO, M. Iniciativas públicas de redução da violência escolar no Brasil. Cadernos de Pesquisa, v. 115, p. 101-138, 2002. https://doi.org/10.1590/S0100-15742002000100004

HOFF, K. E.; REESE-WEBER, M.; SCHNEIDER, W. J.; STAGG, J. W. The association between high status positions and aggressive behaviour in early adolescence. Journal of School Psychology, v. 47, p. 395-426, 2009. https://doi. org/10.1016/j.jsp.2009.07.003 
MARTÍN ÁLVAREZ, A.; OROPEZA, V. de la T.. La interacción transnacional de la sociedad civil centroamericana frente a la violencia juvenil. América Latina Hoy, v. 50, p. 89-102, 2008.

MORENO, D.; NEVES DE JESÚS, S.; MURGUI, S.; MARTÍNEZ, B. Un estudio longitudinal de la reputación social no conformista y la violencia en adolescentes desde la perspectiva de género. Psychological Intervention, v. 21, n. 1, p. 67-75, 2012. https://doi.org/10.5093/in2012v21n1a6

NAVARRO OLIVAS. Factores psicosociales de la agresión escolar: la variable género como factor diferencial. 2009. Tesis (Doctorado en Psicologia) - Universidad de Castilla La Mancha, La Mancha, 2009. Disponible en: https://ruidera. uclm.es/xmlui/handle/10578/1005. Acceso en: 15 jun. 2010.

PARROTT, D. J.; GALLAGHER, K. E. What accounts for heterosexual women's negative emotional responses to lesbians? Examination of traditional gender role beliefs and sexual prejudice. Sex Roles, v. 59, p. 229-239, 2008. https:// doi.org/10.1007/s11199-008-9436-0

PEETS, K.; KIKAS, E. Aggressive strategies and victimization during adolescence: Grade and gender differences, and cross-informant agreement. Aggressive Behavior, v. 32, p. 68-79, 2006. https://doi.org/10.1002/ab.20105

PLATERO, R. La homofobia como elemento clave del acoso escolar homofóbico. Algunas voces desde Rivas Vaciamadrid. Informacion psicológica, v. 94, p. 71-84, 2008.

REIDY, D. E.; SLOAN, C. A.; ZEICHNER, A. Gender role conformity and aggression: Influence of perpetrator and victim conformity on direct physical aggression in women. Personality and Individual Differences, v. 46, p. 231235, 2009. https://doi.org/10.1016/j.paid.2008.10.008

RICHARDSON, D. S.; HAMMOCK, G. S. Social context of human aggression: Are we paying too much attention to gender? Aggression and Violent Behavior, v. 12, p. 417-426, 2007. https://doi.org/10.1016/j.avb.2006.11.001

RITCHIE, J.; LEWIS J. Qualitative research practice. A guide for social science students and researchers. London: Sage, 2003.

SALMIVALLI, C. Bullying and the peer group: A review. Aggression and Violent Behavior, v. 15, p. 112120, 2009. https://doi.org/10.1016/j.avb.2009.08.007

SHUTE, R. H.; OWENS, L. D.; SLEE, P. T. Everyday victimization of adolescent girls by boys: sexual harassment, bullying or aggression? Sex Roles, v. 58, n. 7-8, p. 477-489, 2008. https://doi.org/10.1007/s11199-007-9363-5

VELASCO GÓMEZ, M. J; ÁLVAREZ-GONZÁLEZ, B. Perfiles y percepciones de género en violencia escolar. Revista de Investigación Educativa, v. 33, n. 1, p. 211-231, 2015. https://doi.org/10.6018/rie.33.1.195891

WAASDORP, T. W.; BAKER C. N.; PASKEWICH, B. S; LEFF, S. The association between forms of aggression, leadership, and social status among urban youth. Journal of Youth Adolescence, v. 42, n. 2, p. 263-274, 2013. https://doi.org/10.1007/s10964-012-9837-9

Recibido: 01 Mar 2019

Acepto: 17 Set 2019

Comitê Editorial do Cedes/Coordenação deste número:

Izabel Galvão e Maria Rosa Camargo 